\title{
Effectiveness of Channel-Free Hearing Aid and Noise Desensitisation Training in Auditory Neuropathy Spectrum Disorder - Single Case Study
}

\author{
Sreebha Sreedhar ${ }^{1}$, Nihala K $\mathbf{P}^{2}$, Aleesha $\mathrm{M} \mathrm{H}^{3}$ \\ ${ }^{1}$ Senior Lecturer, National Institute of Speech and Hearing, Trivandrum \\ ${ }^{2}$ Audiologist and Speech Language Pathologist, Maanu Memorial Hospital, Manjeri. \\ ${ }^{3}$ Audiologist and Speech Language Pathologist, Jivadhara Institute of Neurodevelopment and Research, \\ Ernakulum. \\ Corresponding Author: Sreebha Sreedhar
}

\begin{abstract}
Auditory Neuropathy Spectrum Disorder is a rare condition wherein neural transmission through the VIII $^{\text {th }}$ nerve and auditory brainstem is disrupted with intact peripheral hearing. Most frequently reported symptoms by individuals suffering from such conditions include impaired speech discrimination especially in presence of background noise. The aim of this single case study is to emphasize the effectiveness of channel-free technology as a rehabilitative option and to demonstrate the improvement in speech perception in noise with noise desensitisation training. A 24-year-old male patient reported to the National Institute of Speech and Hearing with the complaint of poor speech comprehension. The audiological profile revealed, bilateral moderate sensorineural hearing loss in pure tone audiometry with poor speech discrimination scores, bilateral ' $A$ ' type tympanogram with absent acoustic reflexes, good signal to noise ratio in otoacoustic emissions, and absent Auditory Brainstem Response at $95 \mathrm{dBnHL}$ bilaterally suggestive of auditory neuropathy spectrum disorder in both the ears. As a part of rehabilitation, hearing aids with multiple channels and channel-free technology were tried and better speech discrimination scores were obtained with channel-free technology. In order to address poor speech discrimination in presence of noise, noise desensitisation training was given at different Signal to Noise Ratio with channel-free hearing aids and was found to be effective in improving the speech discrimination scores especially in adverse listening conditions.
\end{abstract}

Keywords: Auditory neuropathy spectrum disorder, Channel free hearing aid, Noise desensitisation.

INTRODUCTION

Auditory neuropathy spectrum disorder (ANSD) is a form of auditory dysfunction in which the peripheral process can be normal, but neural transmission through the VIII $^{\text {th }}$ nerve and auditory brainstem is disrupted. ${ }^{[1]}$ The perceptual consequences of ANSD are distinct from that of cochlear hearing loss and most commonly include distortion of temporal (timing) cues and altered frequency discrimination. This in turn can affect functional hearing, which results in severe impairment of speech perception in the presence of background noise. ${ }^{[1]}$ Discrimination of speech signals in the presence of background noise is a particular problem for listeners with ANSD. ${ }^{[2,3,4]}$ The mechanisms underlying excessive noise effects in ANSD type hearing loss are unclear, although there is psychophysical evidence that auditory signals in ANSD subjects are more affected by simultaneous and non-simultaneous masking than normal listeners. ${ }^{[2,5,6]}$ Although AN/AD can occur in the absence of obvious health problems, the majority of reported cases $(>70 \%)$ have presented specific medical risk factors. ${ }^{[7]} \mathrm{A}$ 
number of different etiologies have been linked with the condition. These include transient neonatal insults (in particular involving anoxia and hyperbilirubinemia), infectious processes (such as mumps and meningitis), and genetic abnormalities including mutations of several genes [OTOF, PMP22, MP2, and NDGRGI] important for peripheral nerve function.

Patients with auditory neuropathy/ dys-synchrony have a range of clinical symptoms. The variability in the clinical features may represent differing degrees of the same pathology or a range of distinct auditory pathway disorders. The sites of lesion include cochlear inner hair cells, the synapse between the inner hair cells and type 1 auditory nerve fibers and the auditory nerve itself. $[8,9,10]$

The two types of ANSD are Postsynaptic ANSD and Presynaptic ANSD. Risk factors include neonatal anoxia, neonatal hyperbilirubinemia, neonatal mechanical ventilation, hypoxia, or both, congenital brain abnormalities, low birth weight, extreme prematurity ( $<28$ weeks), genetics, or family history of ANSD. Clinical Manifestations are disproportionately poor speech recognition abilities for the degree of hearing loss, difficulty hearing in noise, impaired temporal processing, and hearing fluctuation. Not all individuals diagnosed with ANSD experience the same problem.

\section{Clinical findings:}

\section{- Pure tone thresholds \& configuration}

Behavioural detection thresholds are rather widely distributed in both adult and paediatric populations, ranging from normal to profound levels. ${ }^{[9,7]}$ The majority of hearing impairments $82 \%$ were bilateral and symmetrical, with only a few patients having normal hearing in both ears and a unilateral problem.

\section{- Speech identification scores (SIS)}

In ANSD subjects speech perception difficulties are often disproportionate to hearing threshold levels, ${ }^{[11]}$ and they find difficulty hearing in noise. ${ }^{[2,3]}$

\section{- Otoacoustic emissions (OAE)}

Conventionally recorded distortion product and transient OAEs are usually normal or near-normal in individuals with ANSD. OAEs may be present initially and disappear over time in individuals with ANSD. ${ }^{[11]}$ Loss of OAEs, however, does not reflect the change in auditory function or signal conversion of ANSD to typical sensorineural hearing loss.

\section{- Middle ear muscle reflexes (MEMR)}

The majority of ANSD patients have absent acoustic reflex, with about $20 \%$ having an atypically (elevated) acoustic reflex both ipsilaterally and contralaterally.

\section{- Auditory evoked potentials}

Individuals with ANSD have a significantly impaired auditory brainstem response (ABR). Recordings might appear as

1) A flat $A B R$

2) Presence of early peaks i.e., waves up to III with an absence of later waves

3) Some subsequent peaks (wave V) are imperfectly synced yet visible only to stimuli at high stimulus intensity. Cochlear microphonics also provides a valid measure of hair cell function. CMs generally remain present in individuals with ANSD despite the loss of OAEs. [11]

\section{Management}

\section{- Amplification}

Traditional

hearing aid amplification, cochlear implants, and total communication emphasising on visual cues are the widely used rehabilitation methods employed in people with ANSD. The majority of the studies suggest that hearing aids are of limited usefulness in individuals with ANSD ${ }^{[3]}$. In this population, there are two basic grounds against amplification. The first is that amplified sound may cause some damage in these ears having normal 
outer hair cell functioning as evidenced from good responses in Otoacoustic emissions (OAEs). The second is that conventional amplification is not designed to compensate for the temporal distortion introduced into the system by the auditory pathway disorder. That is, the listener will simply be presented with a loud, but equally distorted signal. The limited benefit from hearing aids in individuals with ANSD could be attributed to the inappropriate fitting of the device too. Individuals with ANSD require unique speech processing in hearing aids that would compensate for the affected spectral and temporal processing, in addition to amplifying and making the sounds audible.

With the advancement in technology, hearing aids have gone through several modifications especially in preserving the spectral and temporal cues needed for speech perception. The effectiveness of those advancements need to be explored among ANSD group too. Multichannel compression systems can better accommodate variations in hearing threshold and dynamic range by providing differing amounts of gain across channels ${ }^{[12]}$. Specifically, such hearing aids split the incoming signal into different frequency channels with each channel's gain and output controlled by its own set of controls. Each channel may have a different compression architecture which can be controlled by manipulating the compression threshold, compression ratio, and attack/release times independently in each channel or a selected group of channels. Another advance in technology is channelfree hearing aids. In channel-free hearing aids, based on the individual audiogram, a group of frequency gain curves is established. Out level sampling is carried out around 20,000 times per second by the hearing aid. The Synchronizer in the circuit match the input signal with the appropriate frequency gain curve picked for that level. It is assumed that by not splitting the signal into different compression channels, the hearing aid output retains the spectral contrasts that are present in the input speech. Unlike in multi-channel compression systems, channel-free technology refrains from measuring levels of partial signals in the separate frequency bands. Instead, it measures the wideband SPL value preserving the spectral contrast inherent in the signal ${ }^{[13]}$

\section{- Noise desensitisation training}

Difficulty in listening in presence of noise is one of the major complaints reported by individuals with ANSD. Two types of remediation techniques have been employed to improve a person's ability to listen in noisy conditions. These include environmental modification and deficitspecific intervention. Since it is not always possible to make environmental modifications to reduce noise levels, deficitspecific interventions are more feasible in most cases. To deal with such problems, as early as 1971, Katz and Burge advocated the use of noise desensitisation to help such individuals. Noise desensitisation training may be beneficial for individuals who have difficulty listening to speech in the presence of noise. The auditory system's neurophysiology may have changed as a result of noise desensitisation training, which could explain the improvement after training ${ }^{[14]}$ Noise may be avoided from reaching the limbic and autonomic nervous systems, where it would be prevented from being sensed and interfered with the voice signal. Speech in noise desensitisation training addresses the ability of the brain to process speech in the presence of background noise. Specific acoustic aspects of speech (spectrotemporal) can be disrupted by noise. To tackle this issue, strong neurological function is required. Since noise will reduce synchronization and the number of respondent neurons to speech, it causes an effect similar to reducing the effective intensity of the signal. Hence, the aim of this single case study is to emphasize the effectiveness of channel-free technology as a rehabilitative option and to demonstrate the improvement in speech 
perception in noise with noise desensitisation training among individuals with ANSD.

\section{CASE REPORT}

A patient reported to the National Institute of speech and hearing, Trivandrum with the complaint of reduced hearing and poor speech comprehension especially in the presence of noise for 5 years.

\section{Medical history}

The reports from Medical College Trivandrum reveal (?) Mitochondrial cytopathy and is under medication for the same since 2015. Reports on 5/2/2015 reveal concomitant squint and bilateral lower motor neuron facial palsy. Motor Axonal Neuropathy was diagnosed in the nerve conduction study.

\section{Audiological profile}

The reports on $(29 / 6 / 2018)$ revealed bilateral moderate sensorineural hearing loss (PTA Rt: 43.3dBHL Lt: 46.6dBHL) with poor speech discrimination scores bilaterally. Tympanometry done revealed bilateral ' $A$ ' type tympanogram with absent acoustic reflexes and OAE results showed bilateral good emissions (Rt: 97\% Lt: 98\%) with ABR absent bilaterally at $95 \mathrm{dBnHL}$ suggestive of Auditory Neuropathy Spectrum Disorder. Hence the client was recommended to use speech reading activities at home and also for a hearing aid trial to check the benefit from hearing aid.

\section{Hearing Aid Trial}

An informed consent was obtained from the patient before starting the hearing aid trial. Receiver in the canal (RIC) hearing aids was chosen for the trial. RIC hearing aids having multi channel ( 8 channel) and channel free technology were included in the trial. Both the hearing aids were programmed using the NAL NL2 prescriptive formula. A low-frequency 10 $\mathrm{dB}$ cut was also given for both the hearing aids with a dome with a large vent. The Sound-field thresholds were measured using warble tones in an audiometric test room using GSI Audiostar Pro clinical audiometer and sound field speakers. The results were plotted on a modified Mueller and KillionSII audiogram.

\begin{tabular}{|l|l|l|l|l|l|l|}
\hline Frequency(Hz) & 250 & 500 & 1000 & 2000 & 4000 & SAT \\
\hline Unaided(dB) & 35 & 65 & 55 & 30 & 30 & 35 \\
\hline $\begin{array}{l}\text { Aided(dB) } \\
\text { Channel-free } \\
\text { hearing aid }\end{array}$ & 30 & 40 & 30 & 20 & 15 & 25 \\
\hline $\begin{array}{l}\text { Aided(dB) } \\
\text { Multi-channel } \\
\text { hearing aid }\end{array}$ & 35 & 50 & 45 & 25 & 25 & 30 \\
\hline
\end{tabular}

Also speech discrimination scores were obtained in free field at MCL and +10 dB SNR.

\begin{tabular}{|l|l|l|}
\hline & SDS & SDS at +10dBSNR \\
\hline Unaided & $40 \%$ & $20 \%$ \\
\hline $\begin{array}{l}\text { Aided(dB) } \\
\text { Channel-free hearing aid }\end{array}$ & $65 \%$ & $55 \%$ \\
\hline $\begin{array}{l}\text { Aided(dB) } \\
\text { Multi-channel hearing aid }\end{array}$ & $50 \%$ & $30 \%$ \\
\hline
\end{tabular}

Following the formal assessment, the client was asked to perceptually judge the benefit from both the hearing aids informally. Both the formal and informal assessment revealed better performance with a channel-free hearing aid. Hence the client has been advised to have a trial period of one week with hearing aid at home to assess the hearing aid benefit in an everyday listening environment and he reported benefit after the trial.

\section{Noise Desensitisation training}

In order to address speech discrimination issues in noise, noise desensitisation training was given at different SNR levels along with Channelfree hearing aids. PB words were recorded and mixed with noise using Adobe audition software and instructed the client to practice 20 min every day with a hearing aid for 3 weeks. Each week the client has been assessed and the gain of white noise was increased from $5 \mathrm{~dB}$ to $15 \mathrm{~dB}$ subsequently after each assessment. In order to reduce redundancy different sets of $\mathrm{PB}$ word lists were given for reassessment in noise. 


\begin{tabular}{|l|l|l|l|l|}
\hline Before training with hearing aid & \multicolumn{3}{|l|}{ SDS(\%) at +10dB SNR } & SDS(\%) at 0dBSNR \\
\hline & $\begin{array}{l}\text { I Session } \\
\text { (PB words) }\end{array}$ & $\begin{array}{l}\text { II Session } \\
\text { (PB words) }\end{array}$ & $\begin{array}{l}\text { III Session } \\
\text { (Mono syllables) }\end{array}$ & $\begin{array}{l}\text { III Session } \\
\text { (Mono syllables) }\end{array}$ \\
\hline $55 \%$ & $75 \%$ & $85 \%$ & $85 \%$ & $70 \%$ \\
\hline
\end{tabular}

\section{DISCUSSION}

The channel-free hearing aid was found to be more beneficial for this client. Similar findings were reported by Prabhu \& Barman (2016). ANSD patients have impaired temporal perception ${ }^{[15]}$, spectral smearing and abnormal spectral integration. The multichannel signal processing results in spectral smearing that may further deteriorate temporal and spectral processing leading to poor speech perception. ${ }^{[16]}$ This could be one of the probable reasons for the reported lack of hearing aid benefit among individuals with ANSD $^{[17]}$. Introduction of Channel-free technology that processes signal as a whole without splitting the signal in terms of its frequency components helped to overcome the adverse effect of multichannel compression on the perception of spectral contrasts in speech. ${ }^{[18,13]}$ Thus channel free hearing aids preserve the spectral contrasts in the incoming speech signal as it does not involve splitting signals to different channels. Thus such a technology enhances spectral resolution, temporal resolution and overall sound quality compared with the multi channel processing ${ }^{[13]}$.

Low-frequency cut given also improves the speech clarity by reducing the upward spread of masking ${ }^{[19]}$. They claimed that people with ANSD experience speech primarily through high--frequency information. Low frequencies, on the other hand, are enhanced more with traditional hearing aid amplification. As the majority of patients diagnosed as having ANSD have a reverse sloping or peaked audiogram pattern, the amplification provided in the low frequency region can have an adverse effect in speech perception. When compared to typical behind-the-ear (BTE) hearing aids, receiver in the canal (RIC) hearing aids are said to improve speech clarity. ${ }^{[19]}$. That could be attributed to the fact that the hearing aid users often experience a more natural and clear sound quality when the ear canal is open. Also the use of an open dome fitting reduces the risk of occlusion felt by the hearing aid users. As a result, RIC hearing aids with an open dome provide sufficient venting to relive 0 low frequencies. The inherent benefits of an open-fit RIC hearing aid combined with a low-cut hearing aid modification may enhance sound clarity, which may benefit people with ANSD.

The improvement in speech discrimination scores after noise desensitisation training could be attributed to neurophysiological changes in the auditory system which could account for the improvement following training ${ }^{[14]}$. Speech in noise desensitisation training addresses the brain's ability to process speech in background noise as the noise can disturb specific acoustic characteristics of speech. The adverse signal that is the noise would reduce the neuronal synchronization and the number of respondent neurons to speech, thereby creating an effect similar to reducing effective intensity of signal. Repeated and gradual exposure of sound reduces the aversive effect of sound in individuals by reducing the response of the 2autonomic nervous system and limbic system $^{[1]}$.

\section{CONCLUSION}

This study highlights the auditory outcomes of an adult with an auditory neuropathy spectrum disorder. From the results, a channel-free hearing aid can be considered as effective management for a person with ANSD. Noise desensitisation training has also helped in improving speech in noise perception. However, the study has to be conducted in a large sample size in order to establish effectiveness of Channelfree technology \& noise desensitisation training among individuals with ANSD. More sessions of noise desensitisation with 
patients coming to the institute would have brought a much clearer picture of speech perception in noise and using multi-talker speech babble instead of white noise would have resulted in more benefit.

\section{Acknowledgement: None}

\section{Conflict of Interest: None}

\section{Source of Funding: None}

\section{REFERENCES}

1. Rance, G., 2005. Auditory Neuropathy/Dys-synchrony and Its Perceptual Consequences. Trends in Amplification, 9(1), pp.1-43.

2. Kraus, N., Bradlow, A., Cheatham, M., Cunningham, J., King, C., Koch, D., Nicol, T., McGee3, T., Stein, L. and Wright, B., 2000. Consequences of neural asynchrony: A case of auditory neuropathy. Journal of the Association for Research in Otolaryngology, 1(1), pp.3345.

3. Rance, G., Barker, E., Mok, M., Dowell, R., Rincon, A. and Garratt, R., 2007. Speech Perception in Noise for Children with Auditory Neuropathy/DysSynchrony Type Hearing Loss. Ear \& Hearing, 28(3), pp.351-360.

4. Shallop, J., 2002. Auditory Neuropathy/Dys-Synchrony in Adults and Children. Seminars in Hearing, 23(3), pp.215-224.

5. Vinay and Moore, B., 2007. Ten(HL)-test results and psychophysical tuning curves for subjects with auditory neuropathy. International Journal of Audiology, 46(1), pp.39-46.

6. Zeng, F., Kong, Y., Michalewski, H. and Starr, A., 2005. Perceptual Consequences of Disrupted Auditory Nerve Activity. Journal of Neurophysiology, 93(6), pp.3050-3063.

7. Sininger, Y.S. and Oba, S. 2001. Patients with auditory neuropathy: Who are they and what can they hear? In Y.S. Sininger \& A. Starr (Eds.), Auditory Neuropathy. pp. 15-36.

8. Starr, A., Mcpherson, D., Patterson, J., Don, M., Luxford, W., Shannon, R.,
Sininger, Y., Tonakawa, L. And Waring, M., 1991. Absence Of Both Auditory Evoked Potentials And Auditory Percepts Dependent On Timing Cues. Brain, 114(3), Pp.1157-1180.

9. Rance, G., Beer, D., Cone-Wesson, B., Shepherd, R., Dowell, R., King, A., Rickards, F. and Clark, G., 1999. Clinical Findings for a Group of Infants and Young Children with Auditory Neuropathy. Ear and Hearing, 20(3), pp.238-252.

10. Amatuzzi, M., Northrop, C., Liberman, M., Thornton, A., Halpin, C., Herrmann, B., Pinto, L., Saenz, A., Carranza, A. and Eavey, R., 2001. Selective Inner Hair Cell Loss in Premature Infants and Cochlea Pathological Patterns From Neonatal Intensive Care Unit Autopsies. Archives of Otolaryngology-Head \& Neck Surgery, 127(6), p.629.

11. Starr, A., Picton, T., Sininger, Y., Hood, L. and Berlin, C., 1996. Auditory neuropathy. Brain, 119(3), pp.741-753.

12. Villchur, E., 1973. Signal Processing to Improve Speech Intelligibility in Perceptive Deafness. The Journal of the Acoustical Society of America, 54(1), pp.314-314.

13. Schaub, A. 2009. Enhancing temporal resolution and sound quality: a novel approach to compression. Hear Rev, 16, pp.28-33.

14. Ferre, J. M. 1998. The M3 model for treating CAPD. Central Auditory Processing Disorders: Mostly Management. Boston: Allyn \& Bacon.pp 103-115

15. Rance, G., McKay, C. and Grayden, D., 2004. Perceptual Characterization of Children with Auditory Neuropathy. Ear \& Hearing, 25(1), pp.34-46.

16. Plomp, R., 1988. The negative effect of amplitude compression in multichannel hearing aids in the light of the modulation-transfer function. The Journal of the Acoustical Society of America, 83(6), pp.2322-2327.

17. Prabhu, P. and Barman, A., 2017. Effectiveness of low-cut modified amplification strategy and channel-free hearing aid in individuals with auditory 
Sreebha Sreedhar et.al. Effectiveness of channel-free hearing aid and noise desensitisation training in auditory neuropathy spectrum disorder - single case study.

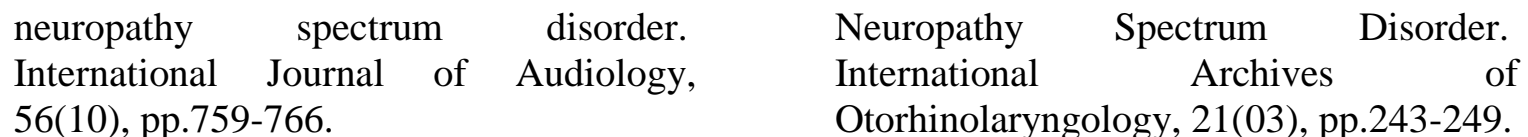

18. Dillon, H., Keidser, G., O'Brien, A. and Silberstein, H., 2003. Sound quality comparisons of advanced hearing aids. The Hearing Journal, 56(4), p.30.

19. Prabhu, P. and Barman, A., 2016. Effectiveness of Low Cut Modified Amplification using Receiver in the Canal Hearing Aid in Individuals with Auditory

How to cite this article: Sreedhar S, Nihala K $\mathrm{P}$, Aleesha M H. Effectiveness of channel-free hearing aid and noise desensitisation training in auditory neuropathy spectrum disorder single case study. Int J Health Sci Res. 2021; 11(10): 162-168. DOI: https://doi.org/10. 52403/ijhsr.20211020 